\title{
Analyse multiéchelle de la diversité génétique des sorghos: compréhension des processus évolutifs pour la conservation in situ
}

Fabrice Sagnard ${ }^{1,2}$

Adeline Barnaud $^{2}$

Monique Deu ${ }^{3}$

Clarisse Barro ${ }^{4}$

Claude Luce ${ }^{5}$

Claire Billot ${ }^{3}$

Jean-François Rami ${ }^{3}$

Sophie Bouchet ${ }^{3}$

Dékoro Dembélé ${ }^{1}$

Virginie Pomiès ${ }^{3}$

Caroline Calatayud ${ }^{3}$

Ronan Rivallan ${ }^{3}$

Hélène Joly ${ }^{2}$

Kirsten Vom Brocke ${ }^{4,5}$

Aboubacar Touré ${ }^{6}$

Jacques Chantereau ${ }^{5}$

Gilles Bezançon ${ }^{7}$

Michel Vaksmann ${ }^{5,6}$

${ }^{1}$ International Crops Research Institute for the Semi-Arid Tropics (ICRISAT),

ICRISAT Kenya,

CO/ILRI,

PO Box 39063

Nairobi

Kenya

$<$ fabrice.sagnard@cirad.fr>

${ }^{2}$ Centre de coopération internationale en recherche agronomique

pour le développement (Cirad),

Unité propre de recherche (UPR) 67

"Gestion des ressources génétiques et dynamiques sociales ",

Unité mixte de recherche' (UMR) 5175-CEFE,

1919, route de Mende,

Montpellier 34293 cedex 5

<adeline.barnaud@cefe.cnrs.fr>

<adelinebarnaud@hotmail.com>

$<$ helene.joly@cefe.cnrs.fr>

${ }^{3}$ Cirad, Unité mixte de recherche (UMR)

"Développement et amélioration

des plantes " (DAP),

Avenue Agropolis,

TA A 96/03,

Montpellier 34398 cedex 5

<monique.deu@cirad.fr>

$<$ claire.billot@cirad.fr>

$<$ jean-francois.rami@cirad.fr>

<sophie.bouchet@cirad.fr>

$<$ virginie.pomies@cirad.fr>

$<$ caroline.calatayud@cirad.fr>

$<$ ronan.rivallan@cirad.fr>n

Tirés à part : F. Sagnard

\begin{abstract}
Résumé
Au Burkina Faso, au Mali, au Niger et à Wanté, 1518 échantillons de sorgho ont été collectés et analysés à l'aide de marqueurs microsatellites. Les paramètres de diversité et de différenciation génétique ont été estimés à différentes échelles spatiales, depuis celle de la variété locale à celle du pays, pour déterminer l'effet des principales forces évolutives sur la diversité génétique du sorgho. Il existe une diversité intra-variétale principalement déterminée par la biologie de la reproduction du sorgho et par les dérives génétiques engendrées par les effectifs limités d'individus lors de l'introduction d'une variété ou de la sélection paysanne de la semence. À l'échelle d'un village, la corrélation entre la diversité des noms vernaculaires et la diversité génétique mesurée par les marqueurs microsatellites est faible. Aucune structuration spatiale n'est observée dans des villages distants de plus de 30 kilomètres soulignant le caractère très local des systèmes semenciers traditionnels. Au Mali et au Niger, une proportion équivalente et supérieure, respectivement, de la diversité génétique du sorgho est observée sur un transect longitudinal qui recoupe essentiellement des divisions ethnolinguistiques, par rapport au transect latitudinal qui recoupe une variabilité agro-écologique plus grande. La diversité des groupes humains explique donc, en interaction avec les conditions environnementales, la diversité génétique du sorgho in situ. Une grande partie de la diversité présente dans la core collection sorgho du Centre de coopération internationale en recherche agronomique pour le développement (Cirad) se retrouve à l'échelle de pays comme le Niger ou le Mali. L'importance de la diversité génétique cultivée par les paysans dans des systèmes agricoles restés traditionnels souligne l'utilité des approches de conservation in situ des ressources génétiques. La conciliation des enjeux de conservation et d'amélioration variétale pourrait être réalisée par un recours plus important au germoplasme local dans des programmes de sélection décentralisés.
\end{abstract}

Mots clés : Afrique occidentale ; conservation des ressources ; marqueur génétique ; ressource génétique ; sorgho.

Thèmes : productions végétales ; ressources naturelles et environnement.

\section{Abstract \\ Multi-scale analysis of sorghum genetic diversity: Understanding the evolutionary processes for in situ conservation}

Using microsatellite genetic markers, we analyzed 1,518 sorghum samples collected at different spatial scales in Burkina Faso, Mali, Niger, and in the village of Wanté, from the landrace to the country scale. Genetic diversity and differentiation parameters were estimated to assess the effects of the main evolutionary processes on sorghum genetic diversity. The genetic variability found within a variety is mainly the result of the sorghum reproduction biology and the genetic drift process caused by the limited number of reproductive individuals at the time of variety introduction into a household or each year when farmers select their seeds. At the village scale, a low correlation is observed between the diversity of vernacular names and the genetic diversity assessed by microsatellites. No spatial genetic structuration is observed among villages more than 30 kilometers apart. This point highlightss that traditional seed systems operate at a very local scale. In Mali, a 
${ }^{4}$ Institut de l'environnement

et des recherches agricoles (Inera),

Programme sorgho,

BP 10, Koudougou

Saria, Burkina Faso

<clarissebk@yahoo.fr>

5 Cirad, Unité propre de recherche (UPR)

"Agrobiodiversité des plantes de savanes"

Avenue Agropolis, TA A-08/01,

34398 Montpellier cedex 5

France

$<$ claude.luce@cirad.fr>

$<$ kirsten.vom_brocke@cirad.fr>

<jacques.chantereau@cirad.fr>

$<$ michel.vaksmann@cirad.fr>

${ }^{6}$ Institut d'économie rurale (IER),

Programme sorgho,

Station de Sotuba, PO Box 258

Bamako, Mali

<acar.toure@ier.ml>

7 Institut de recherche

pour le développement (IRD) Niger,

BP 11416

Niamey, Niger

<bezancon@ird.ne>

\section{Introduction}

Le sorgho (Sorghum bicolor ssp bicolor [L.] Moench.) a été domestiqué il y a plus de 5000 ans, vraisemblablement dans les régions qui correspondent aujourd'hui au sud du Soudan et à l'Éthiopie (Doggett, 1988). Il assure la sécurité alimentaire pour plus de 100 millions d'Africains, en particulier dans les zones semi-arides sahélienne et soudanienne d'Afrique de l'Ouest. Le sorgho est une céréale annuelle, préférentiellement autogame, qui présente une très grande diversité morphologique et génétique, ainsi que des usages multiples. Cette diversité foisonnante décrite par les taxonomistes (Snowden, 1936) a conduit à utiliser une classification simplifiée qui distingue 5 races botaniques principales (guinea, durra, caudatum, bicolor et kafir) et des races intermédiaires partageant des caractères de deux races principales (Harlan et de Wet, 1972).

\section{Agrobiodiversité et diversité génétique}

L'agrobiodiversité peut être appréhendée à différents niveaux d'organisation biologique : de l'agroécosystème à celui de la molécule (Cox et Wood, 1999). La diversité génétique en représente le niveau élémentaire. Son analyse permet d'inférer et d'intégrer des processus évolutifs opérant à différentes échelles spatiotemporelles: des flux de gènes et effets de dérive locaux aux facteurs liés à l'his-

similar proportion of allelic richness is observed along a longitudinal transect and a latitudinal gradient that crosses a larger range of agroclimatic conditions. At the country scale, sorghum exhibits more genetic diversity in Niger than in Mali despite a lower agroclimatic range in Niger. These results demonstrate that the diversity of human groups acts together with the agro-ecological factors to shape the structure of sorghum genetic diversity. An important proportion of the overall genetic diversity present in the Cirad (Centre de coopération internationale en recherche agronomique pour le développement) "core collection" is found in Mali and Niger. The tremendous diversity cultivated by farmers in traditional agroecosystems of Western Africa supports the relevance of in situ approaches for sorghum conservation programs in this region. Both conservation and crop improvement requirements can be achieved through a better use of local germplasm in decentralized breeding programs.

Key words: genetic markers; genetic ressources; resource conservation; sorghum grain; West Africa.

Subjects: natural resources and environment; vegetal productions.

toire de la domestication. En revanche, les zones du génome soumises à la sélection de l'environnement et des paysans (ou des sélectionneurs) ne sont que rarement révélées par les marqueurs génétiques dits neutres. La diversité neutre apparaît cependant corrélée, sous certaines conditions, à la diversité phénotypique (Burstin et Charcosset, 1997). Elle détermine en partie le potentiel adaptatif d'une population ou d'un pool d'individus dans des conditions changeantes. Les marqueurs hypervariables à fort taux de mutation, comme les microsatellites nucléaires (SSR, Simple Sequence Repeats) employés dans notre étude, ont la capacité de révéler de nombreux allèles. La richesse allélique fournit certainement le meilleur indicateur pour identifier des populations qui méritent un effort particulier de conservation (Petit et al., 1998). Les SSR ont des taux de mutation variables selon la taille du motif répété (Sia et al., 1997), le nombre de répétitions (Ellegren, 2004) ou leur proximité de gènes sélectionnés. Aussi, un nombre d'allèles variable à deux loci différents dans deux populations différentes ne peut être considéré comme le seul résultat de processus évolutifs différents. Le faible nombre de marqueurs communs aux études de diversité menées dans plusieurs régions et l'utilisation de méthodes de génotypage difficilement compatibles ont longtemps limité l'intégration de résultats obtenus dans différents projets (Freeman et al., 2005) et, par conséquent, la dimension multiscalaire de notre com- préhension des mécanismes évolutifs de la diversité.

\section{Conservation in situ des ressources génétiques}

Ces dernières décennies ont vu se produire des changements rapides des systèmes de culture d'Afrique subsaharienne et, parfois, une diminution de la diversité des plantes cultivées par les paysans. Au Mali, $60 \%$ des variétés locales (variétés cultivées et reproduites par plusieurs générations de paysans dans une région donnée) de sorgho collectées en 1978 n'ont pas été retrouvées en 1999 (Kouressy, 2002). D'autres ont été introduites par les programmes d'amélioration, les services de vulgarisation, les organisations non gouvernementales (ONG) et le plus souvent à travers des échanges entre paysans ou des achats dans les marchés locaux. Il est souvent difficile de quantifier et d'interpréter ces changements en termes d'érosion génétique.

Si les modes de conservation ex situ et in situ des ressources génétiques apparaissent complémentaires (Swanson et Goeschl, 2000), la conservation in situ manque encore d'une base scientifique solide (Brown, 2000; Brush, 2000). Notamment, notre compréhension de la dynamique in situ de la diversité génétique des plantes cultivées, en relation avec les facteurs environnementaux et anthropiques, est encore limitée. L'intégration de ces facteurs évolutifs, dans le temps et l'espace, est nécessaire à la 
traduction de résultats descriptifs de recherche en outils d'aide à la décision pour les programmes de conservation in situ.

Dans le but de contribuer à la définition des programmes de conservation in situ du sorgho en Afrique de l'Ouest, nous présentons ici une synthèse de l'organisation multiscalaire de la diversité génétique du sorgho à partir de collections réalisées dans quatre pays et d'accessions constituant une core collection à l'échelle de l'aire de répartition mondiale. L'utilisation de marqueurs microsatellites communs et de protocoles de génotypage standardisés a permis l'acquisition de données comparables à plusieurs niveaux d'organisation biologique et l'identification des processus évolutifs principaux à chaque échelle. Nous soulignons également les limites de l'information apportée par les marqueurs microsatellites et faisons des propositions pour mieux concilier les impératifs de conservation et d'amélioration variétale dans les agricultures de subsistance d'Afrique subsaharienne.

\section{Sites, matériel et méthode}

\section{Sites d'étude}

Cette étude est une synthèse des résultats obtenus au Burkina Faso, au Mali, au Niger et dans un village du Nord Cameroun (figure 1), présentant une diversité de systèmes agricoles, de conditions environnementales et d'organisations sociales. Le sorgho y occupe partout une place importante, sinon dominante.

Le Burkina Faso s'étend entre les isohyètes $400 \mathrm{~mm}$ et $1200 \mathrm{~mm}$. Plus de $95 \%$ des variétés cultivées appartiennent à la race guinea (Zongo et al., 1993). Les systèmes de culture à base de sorgho se partagent entre les "champs de case", souvent polyspécifiques et polyvariétaux et les "champs de brousse " où les mélanges variétaux de sorgho sont rares.

Le Mali présente une grande variabilité agroclimatique. La majorité des variétés de sorgho appartient à la race guinea mais les sorghos de la race bicolor et du sous-groupe guinea margaritiferum sont très fréquents dans les zones les plus humides. Le sorgho est généralement cultivé dans des champs monovariétaux à l'exception notable des "champs des

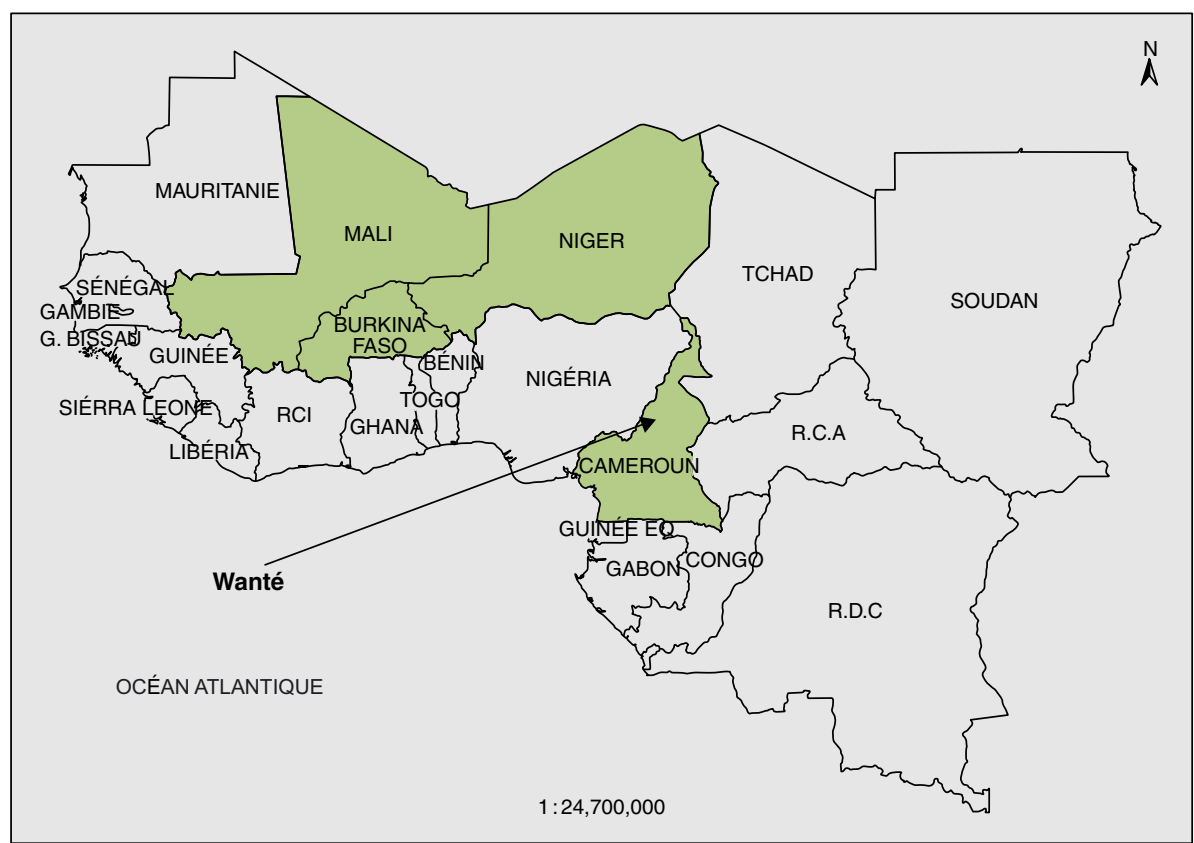

Figure 1. Localisation des sites d'étude.

Figure 1. Location of study sites.

femmes " où les mélanges variétaux sont communs. Les femmes sont d'ailleurs spécialisées dans la culture des types margaritiferum dans le sud et l'ouest du Mali.

La plus grande partie du Niger à l'exception de son extrémité sud-ouest est soumise à un climat sahélien avec une saison pluvieuse excédant rarement 75 jours et des périodes de sécheresses récurrentes. Trois ethnies principales sont engagées dans la culture du sorgho: les Zarma/Songhaïs à l'Ouest, les Haoussas au Centre et les Kanuri à l'extrême-est du pays.

Le village de Wanté est situé au nord du Cameroun ( $\left.8^{\circ} 27^{\prime} \mathrm{N}, 13^{\circ} 18^{\prime} \mathrm{E}\right)$. L'ethnie duupa y pratique une agriculture itinérante sur brûlis. Les céréales sont le pivot des agrosystèmes par leur importance alimentaire, sociale et symbolique. Les Duupa maintiennent une très grande diversité variétale de sorghos cultivés en mélange polyvariétal (Barnaud, 2007).

\section{Matériel collecté et facteurs de structuration analysés}

Les collections de panicules ont été réalisées de manière exhaustive dans chaque village, après discussion avec un groupe représentatif de paysans et remplissage de questionnaires individuels pour cha- que variété collectée. Les panicules ont été prélevées selon les cas, dans le grenier, au champ, ou sur les zones de séchage. Pour l'échantillonnage intravariétal, entre 19 et 22 individus par variété issus de panicules différentes ont été analysés pour les 11 variétés du Cameroun, 24 et 25 individus par variété pour les variétés du Mali (10) et du Burkina Faso (10), respectivement. À l'échelle d'un village, à Wanté, les tailles d'échantillonnages variaient de 4 à 22 individus par variété (289 individus pour les 19 variétés analysées au total). Pour l'analyse intervariétale au Mali (418 variétés dans 60 villages) et au Niger (472 variétés dans 76 villages), une variété était représentée par un seul descendant d'une panicule. Les variétés des villages situés le long de transects latitudinal et longitudinal au Mali ont été regroupées pour former des unités de comparaison avec des effectifs de 50 et 69 individus respectivement.

\section{Extraction d'ADN et génotypage de marqueurs microsatellites nucléaires}

L'ADN a été extrait à partir de feuilles prélevées sur des plantules âgées de 3 à 4 semaines. Les protocoles d'extraction utilisés sont détaillés ailleurs (Barnaud 
Tableau 1. Diversité génétique de variétés de sorghos collectées au Burkina Faso, au Mali et à Wanté au Cameroun.

Table 1. Genetic diversity of sorghums collected in Burkina Faso, Mali, and in Wanté (Cameroon).

\begin{tabular}{|c|c|c|c|c|c|c|c|c|c|c|c|c|c|}
\hline \multirow[t]{2}{*}{ Site } & \multirow{2}{*}{$\begin{array}{c}\text { Type } \\
\text { botanique }\end{array}$} & \multirow[t]{2}{*}{ Caractéristiques } & \multirow{2}{*}{$\begin{array}{l}\text { Nb. } \\
\text { var }\end{array}$} & \multirow{2}{*}{$\begin{array}{l}\text { Nb. } \\
\text { loci }\end{array}$} & \multicolumn{3}{|c|}{$\mathbf{P}$} & \multicolumn{3}{|c|}{$\mathrm{He}$} & \multicolumn{3}{|c|}{ As } \\
\hline & & & & & Med & Min & Max & Med & Min & Max & Med & Min & Max \\
\hline $\begin{array}{l}\text { Burkina } \\
\text { Faso }\end{array}$ & Guinea & & 10 & 12 & 0,83 & 0,42 & 1,00 & 0,36 & 0,08 & 0,46 & 2,67 & 1,83 & 3,42 \\
\hline Mali & $\begin{array}{l}\text { Guinea } \\
\text { margaritiferum }\end{array}$ & $\begin{array}{l}\text { Champs de } \\
\text { femmes }\end{array}$ & 3 & 11 & 0,09 & 0,09 & 0,63 & 0,07 & 0,02 & 0,31 & 1,9 & 1,27 & 2,63 \\
\hline Mali & Guinea & Var. commerciale & 1 & 11 & 0,27 & - & - & 0,08 & - & - & 1,63 & - & - \\
\hline Mali & Guinea & & 6 & 11 & 0,82 & 0,64 & 0,82 & 0,31 & 0,28 & 0,34 & 2,82 & 2,36 & 3,45 \\
\hline Cameroun & Guinea & & 6 & 11 & 0,73 & 0,64 & 0,82 & 0,35 & 0,33 & 0,39 & 3,55 & 3,27 & 3,82 \\
\hline Cameroun & Durra bicolor & Cleistogamie & 1 & 11 & 0,36 & - & - & 0,09 & - & - & 1,64 & - & - \\
\hline Cameroun & Autres & & 4 & 11 & 0,29 & 0,2 & 0,4 & 0,73 & 0,55 & 0,91 & 2,95 & 2,64 & 3 \\
\hline
\end{tabular}

$\mathrm{P}$ : fréquence de loci polymorphes au seuil de $5 \%$; He : diversité génétique ; As : nombre moyen d'allèles par locus. La médiane (Med), le minimum (Min) et le maximum (Max) sont calculés pour chaque groupe de variétés.

et al., 2007). Le génotypage microsatellite a été réalisé sur séquenceur automatique LiCor, à l'exception des analyses faites sur le matériel burkinabé selon la technique d'électrophorèse sur gel de polyacrylamide. Des témoins communs à chaque étude ont été intégrés aux gels, assurant la compatibilité des données produites dans différents projets.

\section{Analyse de la diversité génétique}

La fréquence de loci polymorphes au seuil de $95 \%(\mathrm{P})$, l'hétérozygotie attendue à l'équilibre d'Hardy-Weinberg $(\mathrm{He})$ et le nombre moyen d'allèles par locus (As) ont été estimés pour chaque variété à l'aide du logiciel GENETIX (Belkir et al., 2002) sur l'ensemble des loci analysés. La différenciation génétique mesurée par l'indice $\mathrm{F}_{\mathrm{st}}$ a été calculée selon la méthode de Weir et Cockeram (1984) entre chaque paire de variétés au Burkina Faso, au Mali et au Cameroun, en prenant en compte les variétés d'une même étude. Pour chaque unité d'analyse (variété, village, groupe de villages voisins, transects, pays), et pour les loci communs avec les données de la collection de référence Cirad, la richesse allélique corrigée par la méthode de raréfaction des allèles (Petit et al.,1998) a été rapportée à la richesse allélique d'un échantillon de taille équivalente dans la collection de référence à l'aide du logiciel FSTAT (Goudet, 2001). Le pourcentage de richesse allélique a été calculé de la manière suivante :

\footnotetext{
${ }^{1}$ Voir http://sat.cirad.fr/sat/sorghum_SSR_kit pour plus d'informations.
}

$$
\mathrm{P}_{(\mathrm{Rs})}=100 \times \sum_{\mathrm{i}=1}^{1} \mathrm{Rs}_{\mathrm{Ui}} / \sum_{\mathrm{i}=1}^{1} \mathrm{Rs}_{\mathrm{CCi}}
$$

Où :

$R s_{U i}$ et $R s_{C C i}$ désignent la richesse allélique de deux échantillons de taille identique de l'unité d'analyse $\mathrm{U}$ et de la core collection $\mathrm{CC}$ au locus i respectivement. Sur les données de Wanté, une matrice de dissimilarité a été calculée en se basant sur le nombre moyen d'allèles partagés entre deux individus. Une analyse factorielle sur tableau de dissimilarité (AFTD) a ensuite été réalisée à l'aide du logiciel DARWIN (Perrier et al., 2003).

\section{Résultats et discussion}

\section{Variétés locales, " variétés-populations "}

La diversité génétique intra-variétale présente dans les variétés de sorgho analysées est très variable pour tous les paramètres mesurés (tableau 1). Le taux de locus polymorphe $(\mathrm{P})$ varie de 9 à $100 \%$ l'hétérozygotie attendue (He) de 0,07 à 0,46 et le nombre moyen d'allèles par locus de 1,27 à 3,82. Les variétés les moins diverses sont : i) CSM63E au Mali, une variété locale épurée vendue par les organisations paysannes et collectée une année après son introduction ; ii) See gooriya, une variété durra-bicolor dont les glumes recouvrantes au stade de la floraison assurent une quasi-cléistogamie ; iii) les variétés guinea margaritiferum qui sont cultivées sur de faibles surfaces dans les champs de femmes.
Au Burkina Faso, au Mali et à Wanté, la différenciation génétique moyenne entre les variétés collectées est de 0,37. Les valeurs de $\mathrm{F}_{\text {st }}$ par paire de populations sont très élevées, y compris pour des variétés cultivées dans le même village à Wanté ou dans le même champ à Doubasso au Mali (0,42 entre deux guinea margaritiferum à Doubasso). Les $\mathrm{F}_{\mathrm{st}}$ entre See gooriya et les autres variétés de Wanté varient de 0,57 à 0,75 .

La présence d'une variabilité intravariétale et d'une différenciation génétique variable indique bien le statut de "variété-population " des variétés locales. Elles réagissent ainsi, au même titre que des populations de plantes naturelles aux pressions de sélection, de migration et de dérive, même si l'essentiel de ces forces évolutives est en relation avec des facteurs anthropiques. Les fortes valeurs de $\mathrm{F}_{\mathrm{st}}$ ne peuvent pas être imputées seulement au régime de reproduction préférentiellement autogame du sorgho (sauf dans le cas extrême de la cléistogamie). Les taux d'allofécondation se situent autour de $20 \%$ en moyenne (Djè et al., 2004), ce qui est loin d'être négligeable. De plus, la proximité d'individus de différentes variétés dans les mêmes poquets à Wanté, dans les mêmes parcelles ("champs de femmes") au Mali, et en bordures de parcelles dans les agrosystèmes du Burkina Faso, du Mali et du Niger, devrait favoriser les flux de gènes intervariétaux. Les paysans rapportent indirectement des phénomènes d'introgression en notant des changements de couleur de glumes de leurs variétés ou l'apparition de sorghos de types intermédiaires entre deux variétés. Il semble bien que la dérive génétique joue un rôle prépondé- 
rant. Elle est provoquée par des effectifs reproducteurs limités au moment de l'introduction d'une variété dans une exploitation (généralement à partir d'une ou de quelques panicule(s)) et chaque année au cours de la sélection des semences (Ollitrault et al., 1997).

\section{Structuration génétique des variétés de sorgho à l'échelle d'un village}

L'AFTD (figure 2) montre à la fois une similarité génétique des individus appartenant à la même variété nommée et un regroupement des variétés en partie selon le type botanique. Une analyse bayésienne à l'aide du logiciel STRUCTURE (Pritchard et al., 2000) réalisée sur un jeu de données plus large a montré qu'une organisation de la diversité génétique de 21 variétés nommées par les paysans de Wanté en quatre groupes génétiques était la plus vraisemblable (Barnaud et al., 2007). La richesse allélique des sorghos de Wanté représente 51,5\% de celle observée dans un échantillon de taille identique dans la core collection Cirad. Cette proportion est beaucoup plus importante que pour le village de Pouni-Nord au Burkina Faso $(27,1 \%)$ où seules des variétés guinea sont cultivées. Ces résultats traduisent une faible corrélation entre la diversité vernaculaire et la diversité mesurée par les marqueurs microsatellites. Beaucoup de critères d'identification variétaux utilisés par les paysans reposent sur des caractères morphologiques faiblement polygéniques (par exemple la couleur des grains ou des glumes) qui ont statistiquement peu de chance d'être détectés par des marqueurs neutres à travers l'existence d'un déséquilibre de liaison. Les races botaniques, héritées d'une histoire complexe de domestication, sont un facteur structurant de la diversité génétique à l'échelle de l'aire de répartition (Deu et al., 1994; Deu et al., 2006). La même observation est faite pour les divisions taxonomiques à l'intérieur de la race botanique guinea (Folkertsma et al., 2005). La diversité génétique à l'échelle locale garde cette empreinte historique dans les zones de sympatrie entre différentes races, même dans des systèmes de culture à base de mélanges variétaux comme celui des Duupa. Si des barrières à la reproduction ou des processus de compétition pollinique entre certaines variétés peuvent exister, une contre-sélection par les paysans des individus hors types, issus d'hybridation intervariétale, semble l'explication la plus probable (Barnaud, 2007).

\section{Diversité génétique et systèmes semenciers}

La structuration génétique de variétés appartenant à la même race botanique (guinea au Mali, durra au Niger) cultivées dans des villages de trois divisions

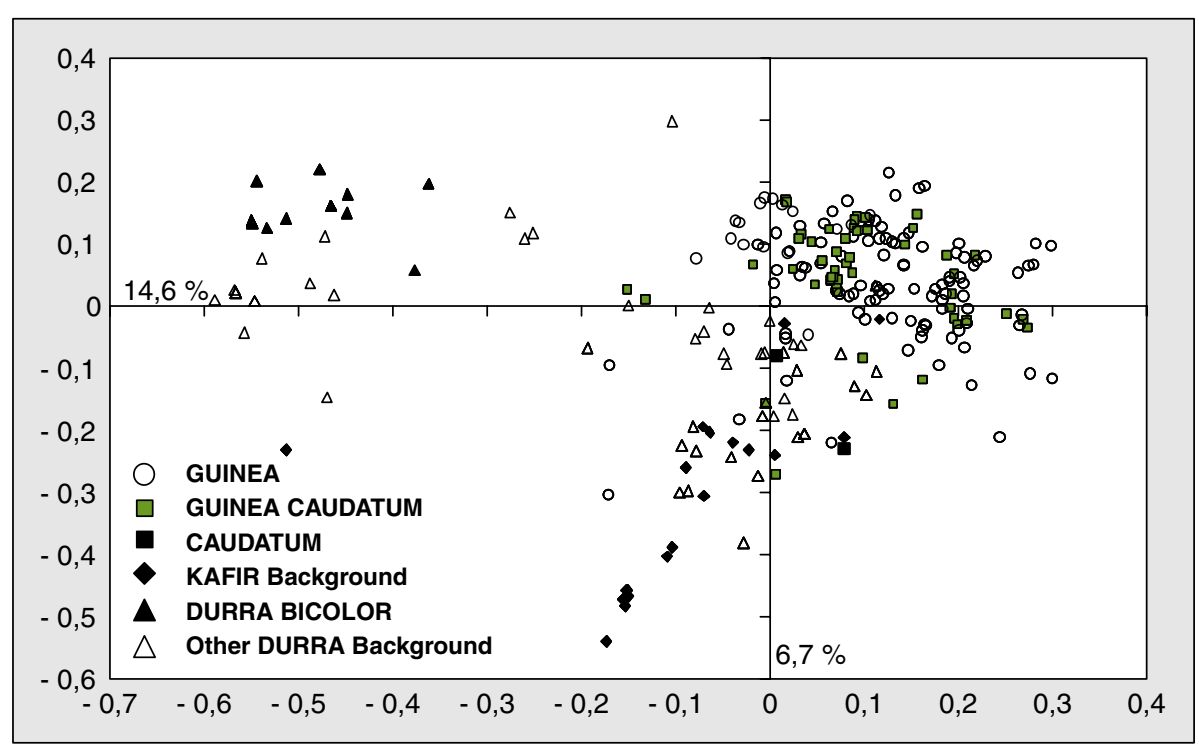

Figure 2. Analyse factorielle sur tableau de dissimilarité (AFTD) de 260 individus appartenant à 19 variétés nommées par les paysans de Wanté (Cameroun).

Figure 2. Principal coordinate analysis of a genetic dissimilarity matrix among 260 sorghums belonging to 19 farmer varieties in Wanté (Cameroon). administratives géographiquement éloignées ne montre pas de tendance d'une organisation spatiale de la diversité (figures 3 et 4). Les villages retenus au sein de la même division administrative étant distants de plusieurs dizaines de kilomètres, les résultats semblent indiquer que les flux de semences restent limités au voisinage direct, dans et à proximité d'un village. Sauf dans le cas de quelques variétés largement diffusées (par exemple CSM63E au Mali), la diffusion limitée des semences peut s'expliquer par une autoproduction largement majoritaire, un système semencier traditionnel privilégiant le voisinage et une forte adaptation des variétés aux contraintes locales de leur milieu de culture.

\section{Facteurs environnementaux et diversité ethnolinguistique}

Les richesses alléliques des sorghos collectés à l'intérieur d'un transect longitudinal entre 12 et 13 degrés de latitude Nord au Mali, et d'un transect latitudinal allant du nord du pays dogon à la frontière avec la Côte d'Ivoire, représente respectivement 60,3 et $61,8 \%$ de la richesse allélique d'un échantillon de taille comparable dans la core collection. Au niveau des pays, ces valeurs atteignent 67,8 et $79 \%$ pour le Mali et le Niger respectivement. Ces résultats indiquent l'importance de la diversité génétique des sorghos cultivés par les paysans dans les agrosystèmes traditionnels proches de centres de diversité (Brush, 2000). Pour le sorgho, la même constatation est faite en Erythrée (Ghebru et al., 2002) et au Bénin (Kayodé et al., 2006). Au Mali, le fait que le transect latidudinal - qui recoupe une variabilité agroécologique plus grande (entre 550 et $1200 \mathrm{~mm}$ de pluviométrie annuelle) - abrite une richesse allélique comparable à celle du transect longitudinal, indique que la structuration de type ethnolinguistique, biogéographique et historique (équivalente dans les deux transects) joue un rôle majeur dans la diversification génétique du sorgho. La même conclusion peut être tirée du fait que les sorghos du Niger possèdent une diversité génétique plus grande que ceux du Mali (les sorghos de décrue de la boucle du Niger n'ont pas été prospectés) malgré des conditions climatiques moins variables. Les sociétés agraires du Niger au cours de leur histoire et grâce à leur positionnement géographique ont accu- 


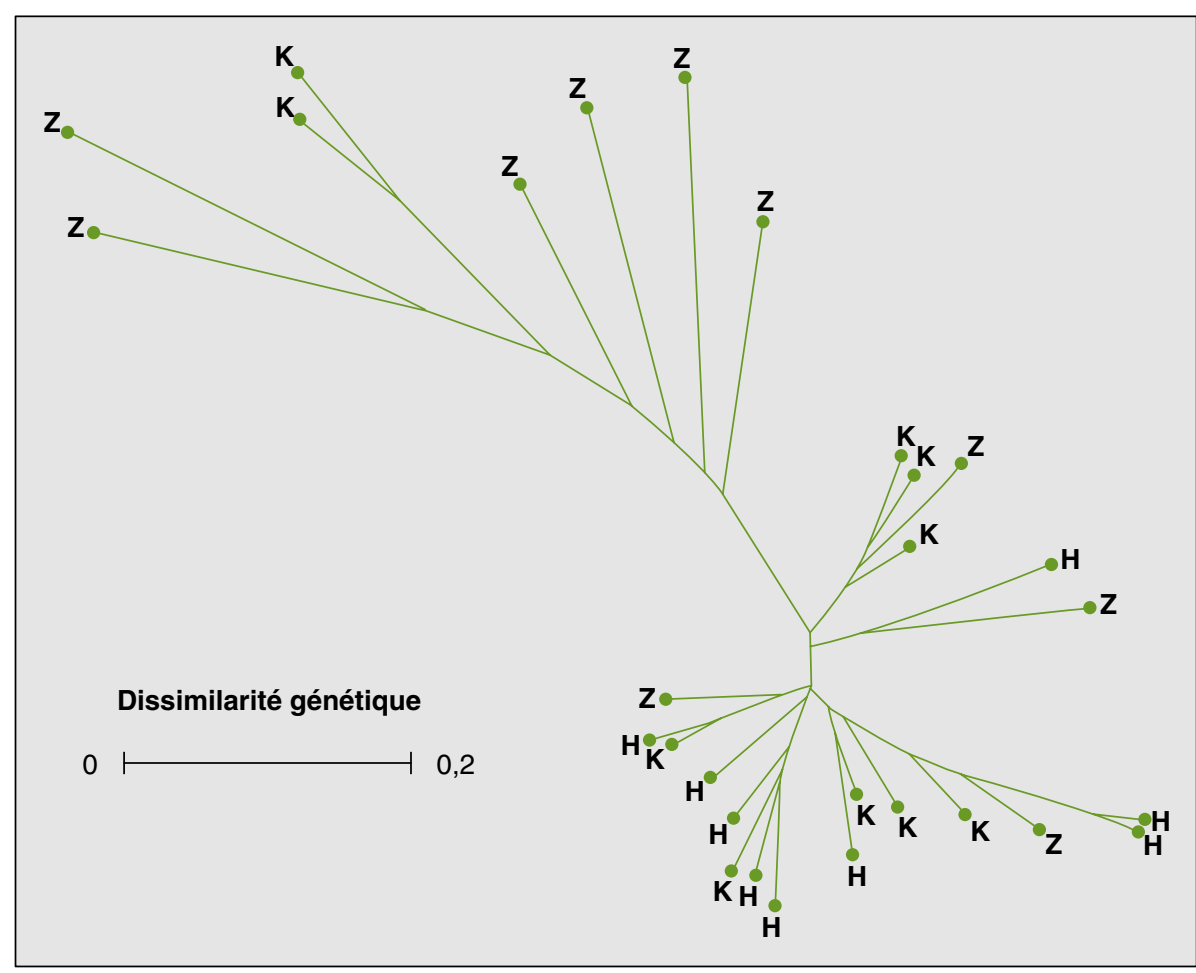

Figure 3. Dendrogramme construit par la méthode Nearest-Joining montrant la structuration génétique de sorghos durra collectés dans trois régions/groupes ethniques du Niger.

Figure 3. Nearest-Joining dendrogram showing the genetic structuration of durra sorghums from three regions/ethnical groups in Niger.

$\mathrm{Z}$ : région Zarma-Songhaï ; $\mathrm{H}$ : région Haoussa ; $\mathrm{K}$ : région Kanuri.

mulé et recombiné les variétés de plusieurs races : durra, caudatum et guinea.

\section{Conclusions}

\section{Interactions entre facteurs environnementaux et anthropiques dans l'évolution des ressources génétiques}

Les facteurs d'évolution de la diversité génétique du sorgho sont de plusieurs ordres. La biologie de la reproduction, la capacité de dispersion du pollen, les systèmes de culture (mélanges de variétés, cultures monovariétales), les pratiques de sélection et d'échanges de semences, mais aussi l'histoire de la domestication et des migrations humaines, interviennent tous dans les structures de la diversité observées aujourd'hui. L'approche génétique infère les processus évolutifs à partir d'une "image " de la diversité contem- tant à l'intérieur d'un village ou entre des villages contigus, l'histoire de la domestication du sorgho et des migrations humaines ont une portée continentale... L'intégration de ces mécanismes évolutifs et la traduction des résultats de la recherche en éléments d'aide à la décision pour la conservation des ressources génétiques nécessitent des allers-retours permanents entre les approches locales et régionales. Les résultats que nous présentons ici, sur la diversité génétique des sorghos en Afrique de l'Ouest, plaident pour une meilleure prise en compte des facteurs sociaux (réseaux d'échanges de semences) et ethnolinguistiques (intégrateurs de la dimension historique) dans la définition des stratégies de conservation in situ. Un réseau de villages situés dans des groupes ethniques différents et soumis à des conditions agroécologiques variables semble être la meilleure stratégie pour représenter la diversité des sorghos au Mali et au Niger. À l'échelle locale, les processus de dérive génétique, dus à des effectifs reproducteurs limités, indiquent l'importance des pratiques de sélection de semences dans la dynamique de la diversité génétique du sorgho. La plupart des paysans exercent une sélection, à la fois conservatrice sur un plan morphologique (recherche d'un idéotype) et source de différenciation génétique (dérive) pour des marqueurs neutres. Enfin, le maintien de l'identité botanique dans des contextes de mélanges polyvariétaux encourage à poursuivre les recherches sur les succès de croisements entre races botaniques et sur la valeur adaptative d'hybrides interraciaux, de manière participative, en milieu paysan.

\section{Conservation de la diversité génétique et amélioration variétale}

Les faibles rendements des variétés locales actuellement cultivées par les paysans en Afrique de l'Ouest ne permettront certainement pas de nourrir une population en forte croissance dans les années à venir. Pour éviter ou limiter la disparition des variétés locales de sorgho, il est donc nécessaire d'améliorer leur rendement et de concilier conservation in situ des ressources génétiques et amélioration variétale. Nos résultats montrent l'importance du réservoir de diversité génétique des sorghos cultivés au Burkina Faso, au Mali, au Niger, et à Wanté. Ces sources de gènes locaux commencent seulement à être exploitées par les programmes d'améliora- 


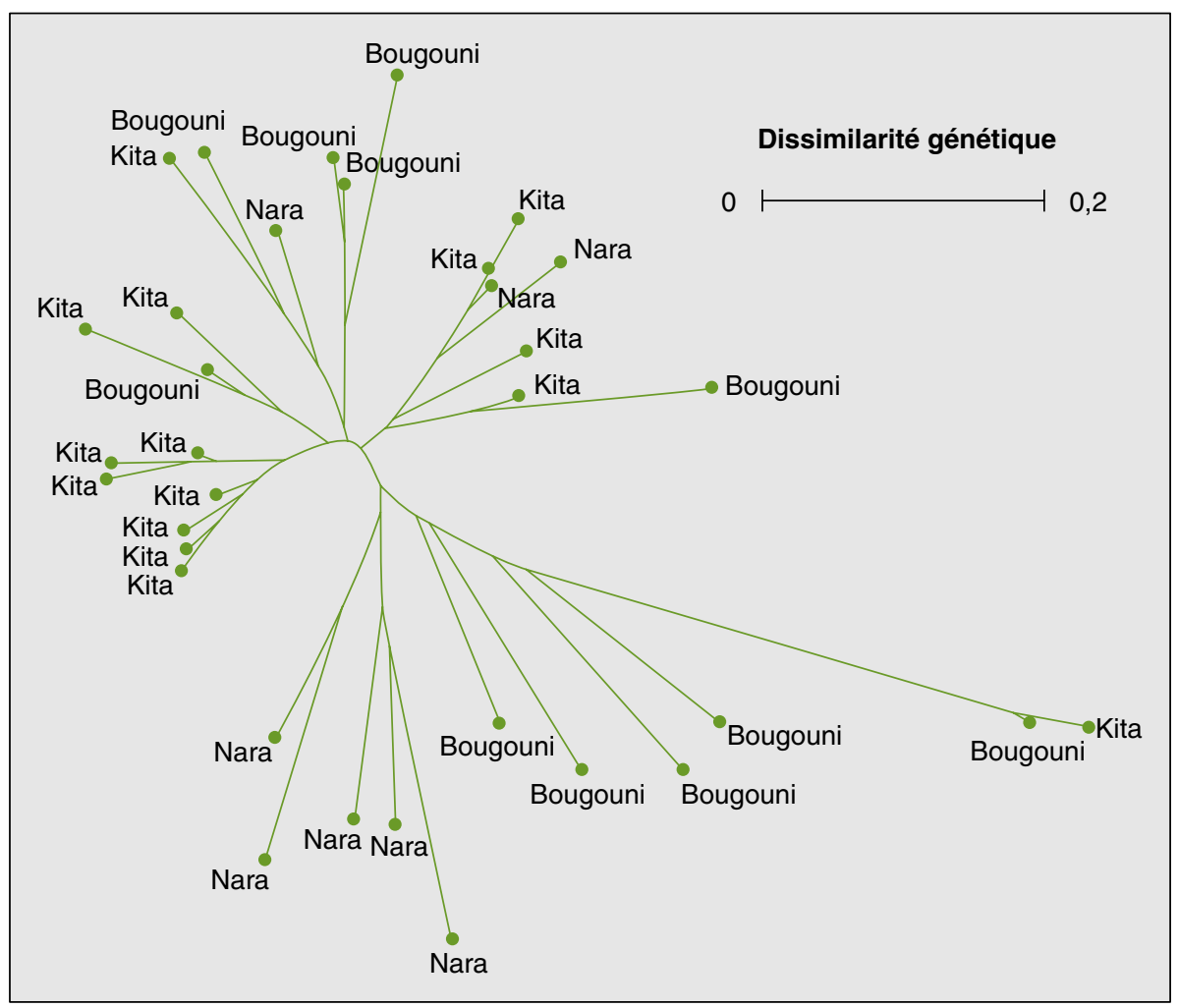

Figure 4. Dendrogramme construit par la méthode Nearest-Joining montrant la structuration génétique de sorghos Guinea guineense collectés dans trois divisions administratives du Mali.

Figure 4. Nearest-Joining dendrogram showing the genetic structuration of Guinea Guineense sorghums from three administrative divisions in Mali.

tion. L'identification et le développement de marqueurs génétiques impliqués dans des caractères d'intérêt agronomique permettront dans l'avenir de mieux lier phénotypes et génotypes, et de rendre plus "fonctionnelles " les analyses de diversité génétique. Les sélectionneurs, quand ils recombinent des gènes locaux dans des variétés améliorées, mènent des programmes de sélection décentralisés et diversifient les structures génétiques des produits de leur sélection (au-delà des lignées pures), jouent donc un rôle moteur, avec les paysans, dans la conservation in situ des ressources génétiques.

\section{Remerciements}

Les résultats utilisés dans cet article proviennent de projets financés par le Fonds français pour l'environnement mondial au Mali et au Burkina Faso, par le programme Biotechnology and Biodiversity Interface de l'United States Agency for International Development (USAID) au Mali, par l'Institut français de la biodiversité au Niger et au Cameroun, et par le Challenge Program
Generation pour les données microsatellites de la core collection Cirad. Nous remercions les équipes techniques de l'International Crops Research Institute for the Semi-Arid Tropics (ICRISAT), de l'Institut d'économie rurale (IER), de l'Institut de recherche pour le développement (IRD) et de l'Institut national de recherche agronomique du Niger (Inran) pour leur participation au travail de terrain. L'appui cartographique de Pierre Sibiry Traoré et de Lassana Touré a également été apprécié. Les commentaires du Pr André Charrier ont permis d'améliorer considérablement

\section{Références}

Barnaud A. Savoirs, pratiques et dynamique de la diversité génétique : le sorgho (Sorghum bicolor ssp. bicolor) chez les Duupa du Nord Cameroun. Thèse de doctorat, université de Montpellier II, 2007.

Barnaud A, Deu M, Garine E, McKey D, Joly HI. Local genetic diversity of sorghum in a village in northern Cameroon: structure and dynamics of landraces. Theor Appl Genet 2007 ; 114 : 237-48. le manuscrit.
Belkhir K, Borsa P, Chikhi L, Raufaste N, Bonhomme F. Genetix 4.04, logiciel sous Windows TM pour la génétique des populations. Montpellier (France) : Laboratoire Génome, Populations, Interactions, CNRS UMR 5000, Université de Montpellier II, 2002.

Brown AHD. The genetic structure of crop landraces and the challenge to conserve them in situ on farms. In : Brush SB, ed. Genes in the field: on-farm conservation of crop diversity. Boca Raton (Etats-Unis): Lewis Publishers, 2000.

Brush SB. The issues of in situ conservation of crop genetic resources. In: Brush SB, ed. Genes in the field: on-farm conservation of crop diversity. Boca Raton (États-Unis) : Lewis Publishers, 2000.

Burstin J, Charcosset A. Relation between phenotypic and marker distances : theoretical and experimental investigations. Heredity $1997 ; 79: 477-83$.

Cox TS, Wood D. The nature and role of crop diversity. In : Wood D, Lenné JM, eds. Agrobiodiversity: characterization, utilization and management. New York (États-Unis): CABI publishing, 1999.

Deu M, Gonzalez-de-Leon D, Glaszmann JC et al. RFLP diversity in cultivated sorghums in relation to racial differentiation. Theor $A p p$ Genet 1994 ; 88 : 838-44.

Deu M, Rattunde F, Chantereau J. A global view of genetic diversity in cultivated sorghum using a core collection. Genome 2006; 49 : 168-80.

Doggett H. Sorghum. $2^{\text {nd }}$ ed. New York (ÉtatsUnis) : Wiley, 1988.

Djè $Y$, Heuertz $M$, Ater $M$, Lefèbre $C$, Vekemans $X$. In situ estimation of outcrossing rate in sorghum landraces using microsatellite markers. Euphytica 2004 ; 138 : 205-12.

Ellegren $\mathrm{H}$. Microsatellites : simple sequences with complex evolution. Nat Rev Genet 2004 ; $5: 435-45$.

Folkertsma RT, Rattunde FHW, Shandra S, Raju GS, Hash CT. The patterns of sorghum diversity of Guinea-race Sorghum bicolor (L.) Moench as revealed with SSR markers. Theor Appl Genet $2005 ; 111$ : 399-409.

Freeman AR, Bradley DG, Nagda S, Gibson JP, Hanotte O. Comination of multiple microsatelitte data sets to investigate genetic diversity and admixture of domestic cattle. Anim Genet $2005 ; 37: 1-9$.

Ghebru B, Schmidt RJ, Bennetzen JL. Genetic diversity of Eritrean sorghum landraces assessed with simple sequence repeat (SSR) markers. Theor Appl Genet 2002 ; 105 : 229-36.

Goudet J. FSTAT, a program to estimate and test gene diversities and fixation indices (version 2.9.3). Lausanne (Suisse) : Université de Lausanne, 2001.

Jarne P, Lagoda PJL. Microsatellites, from molecules to populations and back. Trends Ecol Evol $1996 ; 11$ : 424-9.

Harlan JR, de Wet JMJ. Simplified classification of cultivated sorghum. Crop Sci $1972 ; 12$ : 172-6.

Kayodé APP, Linnemann AR, Nout MJR, Hounhouigan JD, Stomph TJ, Smulders MJM. Diversity and food quality properties of farmers' varieties of sorghum from Bénin. J Sci Food Agr 2006 ; 86 : 1032-9. 
Kouressy M. Étude de la durée du cycle des sorghos locaux du Mali. Comparaison avec la durée des saisons de pluies. Évolution sur les 20 dernières années. DEA, université de Bamako, Mali, 2002.

Ollitrault O, Noyer JL, Chantereau J, Glaszmann JC. Structure génétique et dynamique des variétés traditionnelles de sorgho au Burkina Faso. In : Begic A, ed. Gestion des ressources génétiques de plantes en Afrique des savanes. Bamako (Mali): Institut d'économie rurale (IER); Bureau des ressources génétiques (BRG) ; Solagral, 1997.

Perrier X, Flori A, Bonnot F. Methods for data analysis. In : Hamon $P$, Seguin $M$, Perrier $X$ Glazmann JC, eds. Genetic diversity of cultiva ted tropical plants. Montpellier (France) Science publishers, Inc ; Cirad éditions, 2003.
Petit R, El Mousadik A, Pons O. Identifying populations for conservation on the basis of genetic markers. Conserv Biol 1998 ; 12 : 84455.

Pritchard JK, Stephens M, Donnelly P. Inference of population structure using multilocus genotype data. Genetics $2000 ; 155$ : 945-59.

Schmidt M, Bothma G. Risk assessment for transgenic sorghum in Africa: crop-to-crop gene flow in Sorghum bicolor (L.) Moench. Crop Sci 2006 ; 46 : 790-8.

Sia EA, Kokoska RJ, Dominska M, Greenwell $\mathrm{P}_{\text {, }}$ Petes TD. Microsatellite instability in yeast dependence on repeat unit size and DNA mismatch repair genes. Mol Cell Biol 1997; 17 2851-8.
Snowden JD. The cultivated races of sor ghum. London (Royaume-Uni) : Adlard, 1936.

Swanson T, Goeschl T. Optimal genetic resource conservation : in situ and ex situ. In : Brush SB, ed. Genes in the field: on-farm conservation of crop diversity. Boca Raton (États-Unis) : Lewis Publishers, 2000.

Weir BS, Cockerham CC. Estimating F-statistics for the analysis of population structure. Evolution Int J Org Evolution 1984 ; 38 : 1358-70.

Zongo D, Gouyou PH, Sandmeier M. Genetic variability among sorghum accessions from the Sahelian agroecological region of Burkina Faso. Biodiv Cons $1993 ; 2: 627-36$ 